\title{
A Crash Course on Aging
}

\author{
Giulio Biroli \\ Service de Physique Théorique, Orme des Merisiers, CEA Saclay, 91191 Gif sur \\ Yvette Cedex, France.
}

\begin{abstract}
In these lecture notes I describe some of the main theoretical ideas emerged to explain the aging dynamics. This is meant to be a very short introduction to aging dynamics and no previous knowledge is assumed. I will go through simple examples that allow one to grasp the main results and predictions.
\end{abstract}




\section{Contents}

As the title makes it clear, the aim of these lectures is not to present an exhaustive and complete description of the state of the art in aging dynamics (even less an exhaustive reference list). But rather, its purpose is to present in a nutshell the main theoretical ideas emerged to explain the aging dynamics. For a more complete and deep presentation there are already very good reviews and lectures in the literature 1, 2]. These references are also useful to have an introduction to the experimental results on aging (in particular the ones on memory and rejuvenation effects) which will not be discussed in the following.

The contents of these lectures are:

- Introduction to aging and off-equilibrium thermal relaxation

- A simple example: domain growth in the Ising model

- Mean-Field Theory of Aging

- Activated dynamic and scaling

- The Trap model

- Outstanding issues 


\section{Introduction}

Off-equilibrium dynamics is a very wide and interesting subject. Many works have been devoted to it recently, new interesting theoretical concepts as well as fascinating experimental results have been discovered. Yet, it is certainly fair to say that the overall comprehension is still not complete and certainly many new discoveries (as well as much work) is ahead. There are roughly two different types of off-equilibrium dynamics: driven off-equilibrium dynamics and thermal off-equilibrium relaxation. In the first case the system is driven out of equilibrium by an external stationary force (it may be a shear for a liquid, a voltage for an electron system, etc...) and kept in a stationary off-equilibrium state. The subject of these lectures is the latter type of off-equilibrium dynamics: thermal off-equilibrium relaxation. In this case the system, at equilibrium at time $t_{0}$, is brought out of equilibrium by changing a control parameter $C$ (for example the magnetic field for a magnetic system, or the temperature for a glass-forming liquid, etc...). Subsequently the system, which is in general in contact with a thermal bath, starts to evolve towards the new equilibrium state corresponding to the new value of $C$. However, this may take a long time which actually can become infinite in the thermodynamic limit or just for practical purposes.

From a theoretical point of view this is a clear example in which "more is different". Indeed a system with few degrees of freedom coupled to a thermal bath is going always to equilibrate in a finite time ${ }^{1}$ (which in the worst case scenario and for the majority of systems scales with the number $N$ of the degrees of freedom as $e^{N K}$ where $\mathrm{K}$ is a positive constant). However, when the number degrees of freedom is very large this equilibration time may become infinite for all practical purpose. As in standard equilibrium statistical mechanics the way to deal with this situation is study the system taking the thermodynamic limit from the beginning thus focusing on the evolution of the system on timescales which may be extremely large but do not diverge with the system size.

Let us give just an example before introducing the aging dynamics. Consider a three dimensional Ising ferromagnet at a temperature $T$ larger than the critical temperature $T_{c}$. If one quenches the system from $T$ to $T^{\prime}>T_{c}$ the ferromagnet will relax (i.e. its energy, its local correlation functions, etc..., will relax) on a finite timescale to the new equilibrium state at temperature $T^{\prime}$. However, it has been proven that for $T^{\prime}<T_{C}$ the largest relaxation time scales as $\exp K N^{2 / 3}$, hence, in the thermodynamic limit, the system remains out of equilibrium forever (in the next section we are going to understand in detail the properties of the offequilibrium dynamics in this case). Note that this never-ending off-equilibrium regime is quite general whenever the quench crosses a phase transition. Let us consider another example: the measurement of thermo-remanent magnetization

\footnotetext{
${ }^{1}$ We neglect the cases in which the configuration spaces of the system with few degrees of freedom is broken in disconnected pieces.
} 


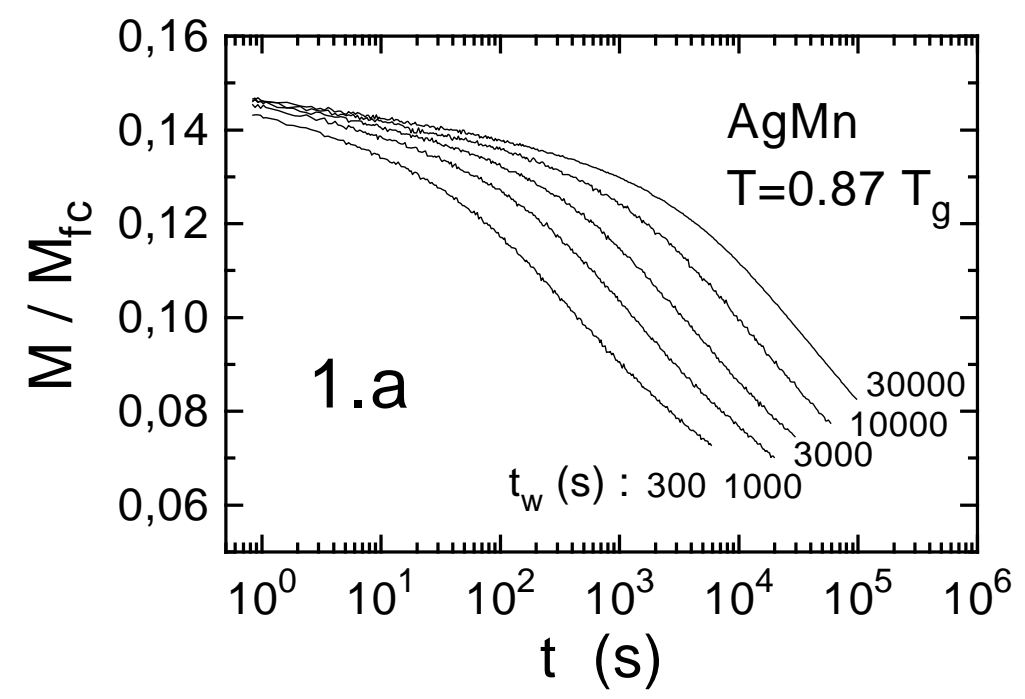

Figure 1: Thermo-remanent magnetization $M$, normalized by the field-cooled value $M_{f c}$, vs. $t(s)\left(\log _{10}\right.$ scale) for a spin-glass $\left(A g: M n_{2.6 \%}\right.$ sample), at $T=$ $9 K=0.87 T_{c}$. The sample has been cooled in a $0.1 \mathrm{Oe}$ field from above $T_{g}=$ $10.4 K$ to $9 \mathrm{~K}$; after waiting $t_{w}$, the field has been cut at $t=0$, and the decaying magnetization recorded (from [2]).

in a spin glass. In this case the system is cooled in a small field from above $T_{c}$ to a temperature below, it then waits in the field for a time $t_{w}$ after which the field is switched off. The subsequent evolution of the magnetization is very peculiar and an example is reported in Fig. 1. After a very rapid fall-off of the magnetization there is a very slow decreasing. A very important feature of these evolutions is that the longer is $t_{w}$ the slower is the relaxation. This behavior is called aging because the system evolution depends on its age $\left(t_{w}\right)$. This means that the typical relaxation timescale for the system is not fixed a priori but is evolving and is fixed by the age of the system itself: older systems relax more slowly.

In order to understand better this point let us again focus on a system with a finite number of degrees of freedom [which therefore has a finite relaxation time]. Generically one expects a similar aging behavior for not too large $t_{w}$. However when the typical relaxation time scale at time $t_{w}$ becomes of the order of the equilibration time all the aging effects are interrupted and the system is characterized by an equilibrium dynamics. This in particularly means that one time quantities like the magnetization, the energy, etc. equal their equilibrium values and, hence, they are time independent; whereas for example the two-time correlation and response functions are invariant under translation of time, i.e. they depend just on the time difference. Finally typical equilibrium relations like fluctuation 
dissipation relations between correlation and response are verified.

Thus, it is clear that aging and the never-ending relaxation discussed before is a collective phenomenon with (at least one) growing correlation length ${ }^{2}$. Indeed one of the theoretical approaches to understand and explain aging dynamics is based on determining what is the growing correlation length, how it grows with time and assuming scaling with respect to this length. Another approach is based on the analytical solution of infinite dimensional (or also infinite number of components) models. In this case an interpretation in terms of a growing lengthscale is at a first sight indirect. Instead the standard physical interpretation that has been developed during the last ten years is in terms of evolution within the energy landscape.

In the following we shall analyze the aging dynamics in the Ising model. This is a good example to start with because it is rather simple and allows one to introduce both approaches which are correct and complementary (in this case at least!). In the following sections I will present more in detail both approaches focusing in particular on their application to disordered systems. Finally I will also briefly present the trap model, that although certainly not a microscopic model, is used often in phenomenological studies and appears as a coarse grained description for some microscopic models.

\section{A simple example: domain growth in the Ising model}

In the following I shall focus on the aging dynamics of the three dimensional Ising model due to a quench from the high disordered temperature phase to a temperature at which, in equilibrium, the system is ferromagnetically ordered. This example is particularly enlightening because it can be analyzed in a simple approximated way (of course much more sophisticated analyzes have been performed). Furthermore all the different theoretical ways to tackle the aging dynamics can be introduced and their physical content can be easily understood. The first thing to do in order to understand what is going on is to look at the results of a numerical simulations. In Fig. 2 there are two snapshots of a $2 \mathrm{~d}$ cut of a $3 \mathrm{~d}$ ferromagnetic Ising model after a quench from the high to the low

\footnotetext{
${ }^{2}$ Why? Well, it is the usual sloppy argument. If all the correlation lengths are bounded during the relaxation then one can roughly divide the system in independent sub-systems for which the relaxation time is bounded by a certain timescale $t_{B}$. Thus the aging has to be interrupted and the system has to relax on a timescales not larger than $t_{B}$ which contradicts the hypothesis of never reaching equilibrium on any finite timescales. In real systems this argument can be used to see that one should have a growing (although not diverging) correlation length even for systems with interrupted aging. Finally, a word of caution, this argument might have problems for system with long range interactions, mean-field models and quench to zero temperature.
} 

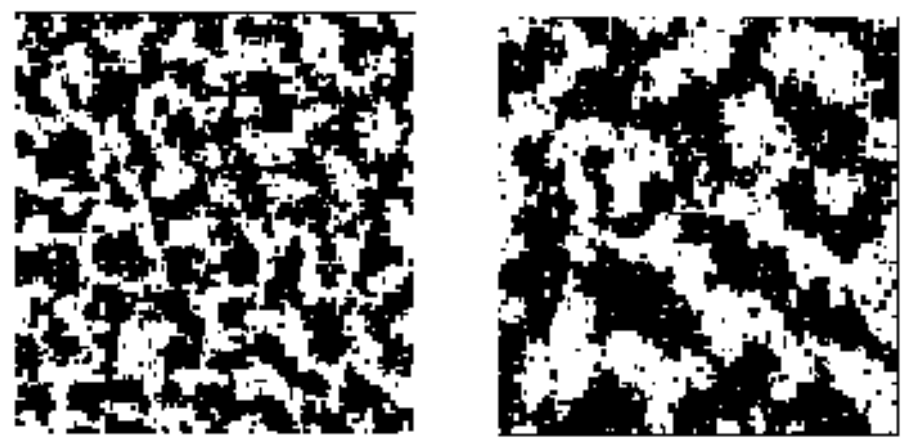

Figure 2: Two snapshots of a $2 \mathrm{~d}$ cut of a $3 \mathrm{~d}$ ferromagnetic Ising model evolving with a Glauber dynamics after a quench at time $t=0$ from high temperature to $T<T_{c}$. Black color is used for minus spins and white for plus spins. On the left the configuration reached after $t_{w}=10^{3}$ MonteCarlo steps. On the right the configuration reached after $t_{w}=10^{5}$ MonteCarlo steps. Statistically the two configurations looks the same after a length rescaling.

temperature phase (black color is used for minus spins and white for plus spins). This figure clearly shows that the systems is trying to separate minus and plus regions in order to gain bulk energy and decrease interface energy: the system is separated in domains that grow in time.

In order to analyze this off-equilibrium dynamic regime it is convenient to set up a continuum description in terms of the coarse-grained magnetization density $\phi(\mathbf{x}, t)$ which obeys a Langevin equation:

$$
\partial_{t} \phi(\mathbf{x}, t)=-\frac{\partial F}{\partial \phi(\mathbf{x}, t)}+\eta(\mathbf{x}, t)
$$

where $\eta(\mathbf{x}, t)$ is a white noise with a variance determined by the temperature $T$

$$
<\eta(\mathbf{x}, t) \eta\left(\mathbf{x}^{\prime}, t^{\prime}\right)>=2 T \delta\left(\mathbf{x}-\mathbf{x}^{\prime}\right) \delta\left(t-t^{\prime}\right)
$$

and $F$ is the standard Ginzburg-Landau free energy

$$
F=\int d \mathbf{x}\left[\frac{1}{2}(\nabla \phi)^{2}-\frac{m^{2}}{2} \phi^{2}+\frac{g}{4} \phi^{4}\right] .
$$

The other important information that one has to provide is the initial condition that we are going to take of the form:

$$
<\phi(\mathbf{x}, 0) \phi\left(\mathbf{x}^{\prime}, 0\right)>=\Delta \delta\left(\mathbf{x}-\mathbf{x}^{\prime}\right) .
$$

Since we are focusing on a system that is in equilibrium at a very high temperature at $t=0$, the correlation length is extremely small and, at our coarse-grained 
level, the magnetization density correlations are just given by (3). At $t=0^{+}$the bath temperature is instantaneously switched to $T<T_{c}$, where $T_{c}$ is the critical temperature of the Ising model. Hence, the system starts to evolve trying to thermalize without never succeeding on any finite time.

In this case the temperature $T$ does not play a very important role, as long as it is smaller than $T_{c}$. Therefore we shall put it equal to zero and we will discuss later what happens for non zero temperature.

As a consequence we have to solve the differential equations:

$$
\partial_{t} \phi(\mathbf{x}, t)=\left(\triangle+m^{2}\right) \phi(\mathbf{x}, t)-g \phi(\mathbf{x}, t)^{3}
$$

with the initial conditions (3). These eqs. correspond to a pure gradient descent in the energy landscape defined by $F$. There are two terms in $F$ : the bulk-energy term $-\frac{m^{2}}{2} \phi^{2}+\frac{g}{4} \phi^{4}$ that is minimized by $\phi= \pm \sqrt{m^{2} / g}$ and the $(\nabla \phi)^{2}$ which is minimized by flat configurations. As a consequence the system during its gradient descent will try to decrease $F$ and this will lead to fatter and flatter regions with a $\phi= \pm \sqrt{m^{2} / g}$ as seen in Fig. 2]

Equations (44) are somewhat simpler than the original stochastic ones but they are still too complicated to be analyzed exactly. Therefore in the following we are going to make use of an approximation à la Hartree that can be justified as a $1 / N$ expansion. It consists simply in replacing the nonlinear term $g \phi(\mathbf{x}, t)^{3}$ by $g<\phi(\mathbf{x}, t)^{2}>\phi(\mathbf{x}, t)$ where $<\cdot>$ means the average over the initial condition ${ }^{3}$. Now the equations read simply:

$$
\partial_{t} \phi(\mathbf{x}, t)=[\triangle+a(t)] \phi(\mathbf{x}, t)
$$

where $a(t)=m^{2}-g<\phi(\mathbf{x}, t)^{2}>$ has to be determined self-consistently (note that since the initial average is translation invariant $<\phi(\mathbf{x}, t)^{2}>$ does not depend on $\mathbf{x}$ ). Eq. (5) can be immediately integrated in Fourier space:

$$
\phi(\mathbf{k}, t)=\phi(\mathbf{k}, 0) \exp \left(-k^{2} t+\int_{0}^{t} a\left(t^{\prime}\right) d t^{\prime}\right)
$$

and the self-consistent equation on $a(t)$ reads:

$$
a(t)=m^{2}-g \Delta \int \frac{d \mathbf{k}}{(2 \pi)^{3}} \exp \left(-2 k^{2} t+2 \int_{0}^{t} a\left(t^{\prime}\right) d t^{\prime}\right)
$$

where eq. (33) has been used to eliminate the initial condition ${ }^{4}$. Since we are interested only to large times and we know that the systems is trying to decrease

\footnotetext{
${ }^{3}$ If one generalizes the field $\phi(\mathbf{x}, t)$ to an $\mathrm{n}$-components field $\phi^{\alpha}(\mathbf{x}, t)$ and $\mathrm{F}$ to $\int d \mathbf{x}\left[\frac{1}{2}(\nabla \vec{\phi})^{2}-\frac{m^{2}}{2} \vec{\phi}^{2}+\frac{g}{4 N}\left(\vec{\phi}^{2}\right)^{2}\right]$ then the equation (4) becomes exact in the infinite $N$ limit. The reason is that in eq. (4) the term $g \phi^{3}$ becomes $g \phi^{\alpha} \vec{\phi}^{2} / N$ and in the infinite $N$ limit the term $\vec{\phi}^{2} / N$ does not fluctuate and is equal to its average.

${ }^{4}$ Some readers can find that this expression is a bit singular at $t=0$. In this case one has to remember that there is an underlying lattice and there is a cut-off in the integration over $k$ corresponding to the inverse of the lattice spacing.
} 
its local energy $-\frac{m^{2}}{2} \phi^{2}+\frac{g}{4} \phi^{4}$ as well the elastic ones $\frac{1}{2}(\nabla \phi)^{2}$ we expect (and it can indeed be shown) that at large times

$$
<\phi^{2}(\mathbf{x}, t)>=\Delta \int \frac{d \mathbf{k}}{(2 \pi)^{3}} \exp \left(-2 k^{2} t+2 \int_{0}^{t} a\left(t^{\prime}\right) d t^{\prime}\right)
$$

converges to a finite value (actually equal to $\mathrm{m}^{2} / \mathrm{g}$ ). But this is possible only if

$$
2 \int_{0}^{t} a\left(t^{\prime}\right) d t^{\prime} \simeq 3 / 2 \log \left(t / t_{0}\right) \quad t_{0}=\left(\Delta m^{2} / g\right)^{2 / 3} / 8 \pi
$$

at large times, i.e. $a(t) \simeq \frac{3}{4 t}$ for $t>>1$. Now that we know the expression of $a$ we can compute the space time correlation function at large times:

$$
<\phi(\mathbf{x}, t) \phi\left(\mathbf{x}^{\prime}, t^{\prime}\right)>=\frac{m^{2}}{g}\left(\frac{4 t t^{\prime}}{\left(t+t^{\prime}\right)^{2}}\right)^{3 / 4} \exp \left(-\frac{\left(\mathbf{x}-\mathbf{x}^{\prime}\right)^{2}}{4\left(t+t^{\prime}\right)}\right)
$$

This expression is very interesting and deserves different remarks. First, for any finite and fixed $\left(\mathbf{x}-\mathbf{x}^{\prime}\right)$, it becomes at large times simply a function of $t^{\prime} / t$. Thus, we have found that the correlation between two points, as far as they could be in space and time, converges to the value $m^{2} / g$ when $t^{\prime}, t \rightarrow \infty$ provided that their distance in space and time is kept fixed when the large time limit is taken, i.e. if one looks to a finite region on a finite time this region will be typically on one of the two state $\pm \sqrt{m^{2} / g}$. On the other hand, for fixed but very large time $t, t^{\prime}$ the magnetization density correlation always fall to zero over distance $\left|\mathbf{x}-\mathbf{x}^{\prime}\right| \propto \sqrt{t+t^{\prime}}$. This means that, at time $t$, the typical size of the regions in the states $\pm \sqrt{m^{2} / g}$ is $L(t) \propto \sqrt{t}$. Furthermore, for fixed $\left|\mathbf{x}-\mathbf{x}^{\prime}\right|$, the magnetization density correlation always falls to zero over a time separation $t-t^{\prime}$ which is larger than $t^{\prime}$ (we assume $t^{\prime}<t$ ). This means that the time it takes to the system to decorrelate from its configuration at time $t^{\prime}$ is of the order of $t^{\prime}$ itself, i.e. the age of the system is the characteristic timescale for the dynamical evolution: the older is the system, the slower is its dynamics.

Thus we recover what discussed in the introduction and plotted in Fig. 2, the systems is decomposed in domains of size $L(t)$ (that is the growing correlation I talked about in the introduction), for which we have found a growth law $L(t) \propto$ $\sqrt{t}$. In the interior of each domain the system is in one of the two state $\pm \sqrt{m^{2} / g}$. However, if one waits a time of the order of the age of the system a point $\mathbf{x}$ has been swept by different domain walls and thus the magnetic correlation has been lost.

In this approximate treatment of this simple model we find the essential ingredient of the coarsening/scaling picture of the aging dynamics. On long time-scales the system is broken up in domains which grow and the dynamics is self-similar provided the length is appropriately rescaled by $L(t)$. This length $L(t)$ is naturally interpreted as the typical size of the domains and its rate of growth can be 
found from general arguments [3]. In this case $L(t) \propto \sqrt{t}$ but the growth rate may be different for different systems [3]. For example, if we consider the Ising model but with a dynamics that conserves the magnetization, for example the Kawasaki dynamics [3, then $L(t) \propto t^{1 / 3}$. However, because of the self-similarity of the dynamics, one generically expects that the correlation function can be written in a scaling form

$$
<\phi(\mathbf{x}, t) \phi\left(\mathbf{x}^{\prime}, t^{\prime}\right)>=f\left(\frac{\left|\mathbf{x}-\mathbf{x}^{\prime}\right|}{L(t)}, \frac{L(t)}{L\left(t^{\prime}\right)}\right)
$$

This is indeed the case in our approximate treatment and we refer to [3, 1] for a more general discussion.

Another way to think about aging and glassy dynamics is the one coming from the solution of mean-field disordered systems very much based on the energy landscape (or more precisely free energy but here it does not matter because $T=0$ ). Although we postpone a more technical discussion to the next section, in the following we shall introduce the main ideas [1, 4. Let us start with some general remarks.

The equation (4) consists in a gradient descent in the energy landscape from a high temperature configuration toward the ground state configurations (that are never reached on finite time scales). During this gradient descent the velocity of the system decreases and tends to zero in the infinite time limit. This rather natural results can be checked noticing that the square of the absolute value of the velocity in the energy landscape $\vec{v}=-\nabla F=-\frac{\delta F}{\delta \phi(\mathbf{x})}$ equals $-\frac{d F}{d t}$. Since on general grounds one expects that one time quantities, in particular $F$, tends toward a well defined value in the infinite time limit (indeed one can check that this is the case within our Hartree approximation) we obtains that $\frac{d F}{d t}$ and the velocity has to tend to zero in the infinite time limit.

Another important quantity other than the velocity that is useful to track the system evolution is the the energy Hessian evaluated at the configuration reached at time $t$, in particular its density of eigenvalues. Since the system is trying to reach the completely stable states $\pm \sqrt{m^{2} / g}$ we expect that the fraction of negative eigenvalues becomes smaller and smaller at large times and vanishes at $t=\infty$. Let us check this assumption within our approximate treatment in which the Hessian is diagonal in Fourier space:

$$
H=\frac{\delta F}{\delta \phi(\mathbf{k}) \delta \phi\left(\mathbf{k}^{\prime}\right)}=\left[\mathbf{k}^{2}-m^{2}+g<\phi(\mathbf{x}, t)^{2}>\right] \delta\left(\mathbf{k}+\mathbf{k}^{\prime}\right) .
$$

Computing the density of states is an easy job and one finds:

$$
\rho_{t}(\lambda)=\frac{1}{4 \pi^{2} N} \sqrt{\lambda+m^{2}-g<\phi(\mathbf{x}, t)^{2}>} .
$$




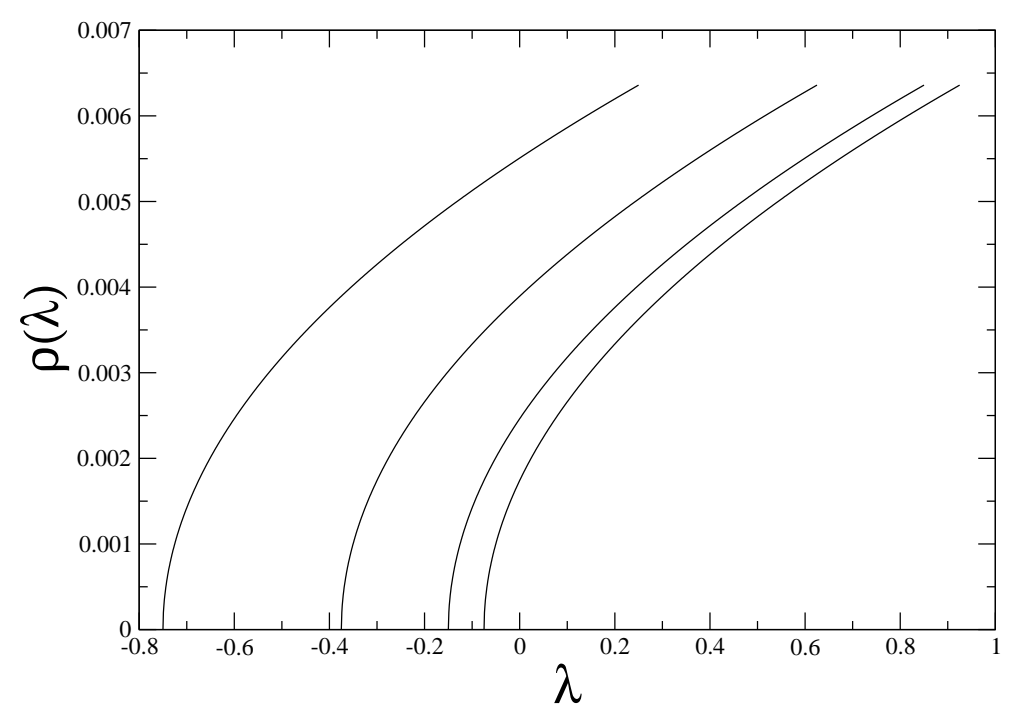

Figure 3: $\rho_{t}(\lambda)$ as a function of $\lambda$ for $t=1,2,5,10$ (from left to right). For simplicity $N$ is put equal to one.

where $N$ is a normalization factor ${ }^{5}$. Since $m^{2}-g<\phi(\mathbf{x}, t)^{2}>=a(t) \simeq 3 / 4 t$ we find that the density of states of the Hessian has at its left edge a square root form starting at a negative value $\lambda_{\min } \simeq-3 / 4 t$ which indeed approaches slowly zero at infinite times, see Fig 3. Finally, the last piece of information that we need is that the velocity always points toward directions corresponding to small eigenvalues of the Hessian. This can be checked noticing that when the energy decreases slower than exponentially to its infinite time value (a quite general behavior in aging system that is true in our case):

$$
\frac{\frac{d^{2} F}{d t^{2}}}{\frac{d F}{d t}}=-2 \frac{\nabla F \cdot H \cdot \nabla F}{(\nabla F)^{2}} \rightarrow 0
$$

when $t \rightarrow \infty$. Hence, decomposing this identity along the eigenvectors of $H$ and calling $v_{\mu}$ the components of $\nabla F$ on the eigenvector $\mu$ we find:

$$
\frac{\sum_{\mu} v_{\mu}^{2} \lambda_{\mu}}{\sum_{\mu} v_{\mu}^{2}} \rightarrow 0
$$

which shows that indeed the velocity always points toward directions corresponding to small (vanishing) eigenvalues of the Hessian.

Putting all these pieces of information together, we obtain an energy landscape picture of aging in which the system makes a gradient descent in the energy

\footnotetext{
${ }^{5}$ Note that since there is an underlying lattice there is also a cutoff in the integration over k. Therefore $N$ is cut-off and lattice dependent. Also $\rho(\lambda)$ is in reality cut-off and lattice dependent except at its left edge where is given by eq. (7).
} 
landscape and at long times it moves slowly and slowly (the absolute value of the velocity is going to zero) along channels with many uphill directions and a low density of downhill directions. The system velocity points towards the directions over which the energy landscape is flatter. The longer is the time the smaller is the fraction of downhill directions and they are more and more flat. Thus it takes longer to the system to move and go downhill. This never-ending descent in the energy landscape dominated by an increasing flatness of the landscape is the main picture behind aging obtained from the exact solution of the dynamics of different mean-field models [1].

Finally, let me comment on how things change if the final temperature after the quench is not zero (but still less than $T_{c}$ ). In this case inside the domains the system has a pseudo-equilibrium dynamics that takes place on a finite timescale. For example, the local spin-spin correlation function will be approximatively a function of $t-t^{\prime}$ for $t-t^{\prime}<<t^{\prime}$ (and $t^{\prime}>>1$ ) that converges to a plateau $m_{i}^{2}$ on a finite timescale where $m_{i}$ is the local magnetization. The aging regime shows up only on timescales such that $t-t^{\prime} \propto t^{\prime}$. It corresponds to the exit from the plateau and can still be described in the same way discussed above.

\section{Disordered systems}

In the following I will discuss in some detail the theoretical approaches introduced to explain the aging dynamics. I will focus mainly on disordered systems for historical reasons and simplicity and I will comment in the conclusion on the generalization to non-disordered glassy systems.

\subsection{Mean-Field Theory of Aging}

The study of the equilibrium and off-equilibrium dynamics of mean-field disordered systems revealed that a very rich and interesting behavior can be found within the mean-field approximation. Generically, it has been found that these systems displays a dynamical transition at a temperature $T_{d}$ at which the relaxation time diverges. A quench to a temperature less than $T_{d}$ leads to an offequilibrium behavior and aging that persist forever if the thermodynamic limit has been taken from the beginning. The way in which the dynamical transition affects the equilibrium dynamics for $T>T_{d}$ is discussed in the Lectures of A. Cavagna [5] and D. R. Reichman [6] and I will not discuss it. I would like just to point out that two quite different behaviors may take place. In mean-field disordered systems having a spin-glass transition, like for example the Sherrington-Kirkpatrick model, the dynamical transition temperature coincides with the temperature at which the equilibrium glass transition takes place. Instead, for systems like the p-spin disordered model, which have an equilibrium transition conjectured to be related to the structural glass transition, the dy- 
namical transition temperature is larger than the equilibrium one. Furthermore, contrary to the first case, the transition has a (partially) first order character since a plateau strictly different from zero emerges in the correlation functions at $T_{d}$, see the left plot in Fig 4

Historically, the mean-field theory of aging has been developed analyzing the
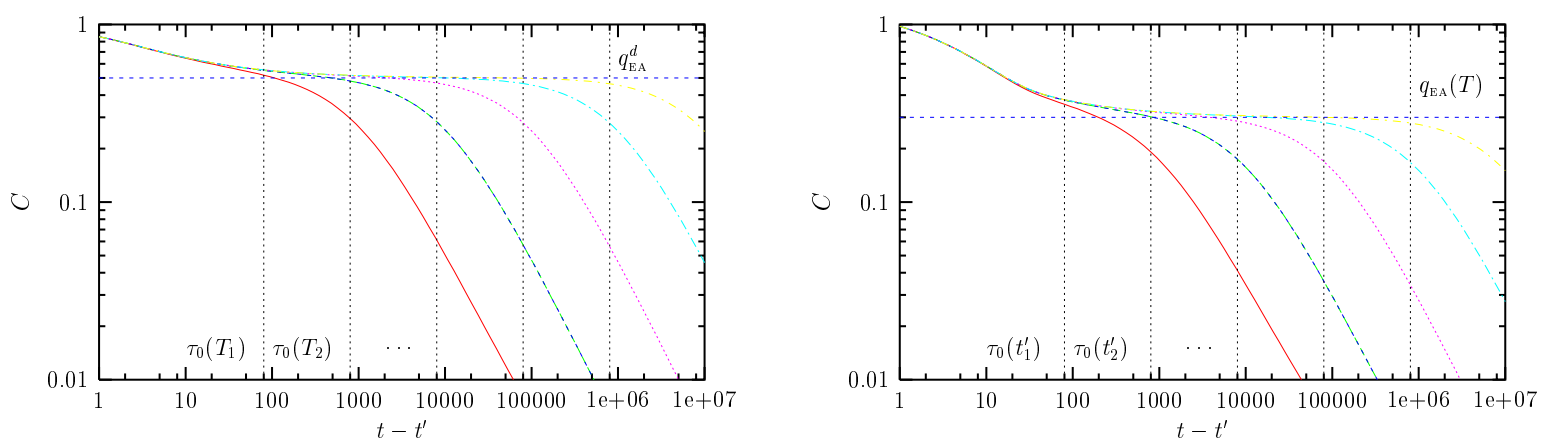

Figure 4: Left: Sketch, for a p-spin model, of the decay of the stationary correlations in the high $T$ phase close to $T_{d}, T_{1}>T_{2}>\ldots$ (from left to right). Right: Sketch, for a p-spin model, of the decay of the aging correlations in the low $T$ phase, at fixed $T<T_{d}, t_{1}^{\prime}<t_{2}^{\prime}<\ldots$ (from left to right).

off-equilibrium dynamics of completely connected models. An example is the p-spins model whose Hamiltonian is

$$
H=\sum_{1 \leq i_{1} \leq \ldots \leq i_{p}} J_{i_{1}, \ldots, i_{p}} s_{i_{1}} \cdots s_{i_{p}}
$$

and the couplings $J_{i_{1}, \ldots, i_{p}}$ are independent Gaussian variables with zero mean and variance $p ! / 2 N^{p-1}$. The dynamics of this systems can be fully analyzed if the degrees of freedom are variables $s_{i}$ subjected a global spherical constraint $\sum_{i=1}^{N} s_{i}^{2}=N$. The usual strategy is to take a Langevin dynamics for these (spherical) models and write down the Schwinger-Dyson equations for the correlation $C$ and response functions $R$ :

$$
C\left(t, t^{\prime}\right)=\frac{1}{N} \sum_{i} \overline{<s_{i}(t) s_{i}\left(t^{\prime}\right)>} \quad R\left(t, t^{\prime}\right)=\frac{1}{N} \sum_{i} \frac{\overline{<s_{i}(t)>}}{h_{i}\left(t^{\prime}\right)},
$$

where $h_{i}\left(t^{\prime}\right)$ is a local magnetic time-dependent field coupled to $s_{i}$ and the overline means the average over disorder. A great simplification due to the completely connected character of these models is that the self-energy can be written as a simple polynomial function of correlation and response. More specifically, the general expression one finds is [1]:

$$
\int_{0}^{+\infty} d t_{1} R_{0}^{-1}\left(t, t_{1}\right) C\left(t_{1}, t^{\prime}\right)-2 T R\left(t^{\prime}, t\right)=\int_{0}^{+\infty} d t_{1} \Sigma_{C}\left(t, t_{1}\right) R\left(t^{\prime}, t_{1}\right)+
$$




$$
\begin{aligned}
& +\int_{0}^{+\infty} d t_{1} \Sigma_{R}\left(t, t_{1}\right) C\left(t_{1}, t^{\prime}\right) \\
\int_{0}^{+\infty} d t_{1} R_{0}^{-1}\left(t, t_{1}\right) R\left(t_{1}, t^{\prime}\right)= & \delta\left(t-t^{\prime}\right)+ \\
& +\int_{0}^{+\infty} d t_{1} \Sigma_{R}\left(t, t_{1}\right) R\left(t_{1}, t^{\prime}\right)
\end{aligned}
$$

where $R_{0}^{-1}$ is the inverse of the bare response function. For example in the case of the p-spin spherical model $R_{0}^{-1}\left(t, t_{1}\right)=\left[\partial_{t}+\mu(t)\right] \delta\left(t-t_{1}\right)$ where $\mu(t)$ is a spherical Lagrange multiplier determined enforcing the spherical constraint $C(t, t)=1$. The self-energies are simple functions of the propagators and read:

$$
\Sigma_{C}\left(t, t^{\prime}\right)=\sum_{n} c_{n}\left[C\left(t, t^{\prime}\right)\right]^{n} \quad \Sigma_{R}\left(t, t^{\prime}\right)=\sum_{n} n c_{n}\left[C\left(t, t^{\prime}\right)\right]^{n-1} R\left(t, t^{\prime}\right)
$$

and the coefficient $c_{n}$ depends on the particular model one is focusing on. For example in the case of the p-spin spherical model all the $c_{n}$ are zero except $c_{p-1}=p / 2$, i.e. $c_{n}=\delta_{n, p-1} p / 2$.

At this point it is easy to understand that it is possible to derive these type of equations not only within the exact solution of completely connected models but also within self-consistent approximate treatment of finite dimensional systems. Indeed in the case of Langevin dynamics one can set up a field theory in which the self-energy is written as a sum of diagrams constructed with the vertices of the theory and using the true propagator as line. The equation (11) is in general equivalent to retain only the first self-consistent diagram and can be often justified in terms as a large $N$ expansion in $1 / N$. This also makes it clear why this the type of eqs. introduced above can be found also for non-disordered systems. More complicated functional forms have been also partially studied in the literature but I will not discuss it further.

There are two methods for analyzing the eqs. (910): a complete solution can be obtained by integrating them numerically but much information can be gained already analytically. In the following I will just make a summary of the main findings.

For $T>T_{d}$ correlations and responses converge at large times toward a time translation invariant regime (TTI), i.e. they depend only on the time difference $t-t^{\prime}$ and furthermore they are related by the fluctuation-dissipation relation $R(\tau)=-C^{\prime}(\tau) / T$. Hence, the system is at equilibrium. Approaching $T_{d}$ the correlation function develops a plateau for discontinuous spin models and takes more and more time to attain the equilibrium TTI regime, see Fig. 4. Instead for $T<T_{d}$ the correlation and response never reach the equilibrium and a TTI regime. In simple cases, for example in the case of the p-spin spherical model, there are two time sectors: a pseudo-equilibrium one that corresponds to taking the limit $t^{\prime} \rightarrow \infty$ with $t-t^{\prime}$ fixed. In this regime the correlation and response become indeed TTI and are related by the fluctuation dissipation relation (as for 
the domain growth discussed in the previous section). The aging regime corresponds to $t \rightarrow \infty$ with $t^{\prime} / t$ fixed in which the correlation and response function become functions of $t^{\prime} / t$. The correlation function equals the Edwards-Anderson parameter $q_{E A}$ for $t^{\prime} / t=1$ and approaches zero in the limit $t^{\prime} / t=0$ (I take $t^{\prime}<t$ ), see Fig 团 Note that in more complicated case the $t^{\prime} / t$ is replaced by $h\left(t^{\prime}\right) / h(t)$ with $h$ a model dependent function. It may also happen, in particular for continuous models that there is an infinite sequence of time sectors, each one of them determined by a ratio $h_{i}\left(t^{\prime}\right) / h_{i}(t)$.

Finally, a striking property of this off-equilibrium regime is that the correlation and response are still related by a fluctuation-dissipation like relation (FDR) in which, however, the bath temperature is replaced by an effective temperature $T_{\text {eff }}$. In the case of different time sectors there is an effective temperature for each one of them. The interpretation of $T_{\text {eff }}$ as an effective temperature has been justified in different papers. Furthermore, it has also been shown that $T_{\text {eff }}$ can be related in some cases to the Parisi function $x(q)$ introduced in the study of the thermodynamics. This topic has generated an enormous amount of interest: many numerical simulations and some experiments have been performed in order to check the existence of this off-equilibrium relation between correlation and response. A very popular way to plot data to check for a generalization of FDR consists in plotting the integrated response $\chi\left(t, t_{w}\right)=\int_{t_{w}}^{t} R\left(t, t^{\prime}\right) d t^{\prime}$ as a function of the correlation $C\left(t, t_{w}\right)$. If the fluctuation dissipation relation $R=-C^{\prime} / T_{\text {eff }}$ is verified (in the limit $t_{w} \rightarrow \infty$ ) one should find a straight line with slope $-1 / T_{\text {eff }}$. In Fig. 5 we plot on the left a typical FDR plot for a system converging to equilibrium. In this case the curves, for different $t_{w}$ evolves toward a straight line. Instead for mean-field glassy systems off-equilibrium a rather different behavior has been found, see the right part of Fig. [5. The dot-dashed line corresponds to a case with an infinite number of timescales and $T_{\text {eff }}$. The continuous line with a breaking point corresponds to a systems, like the p-spin spherical model, with only one $T_{\text {eff }}$. The dotted line corresponds to the domain growth in the Ising model $T_{\text {eff }}=\infty$. The other curves correspond to waiting times far from the asymptotic regime. A detailed discussion about $T_{\text {eff }}$ can be found in [1]. I will just make some other comments in the conclusion.

Finally, let me discuss the physical interpretation of this aging dynamics. As anticipated in the previous section the interpretation that comes from many different analytical and numerical studies is based on a never-ending descent in the (free-)energy landscape ${ }^{6}$. Indeed the aging after quench at zero temperature is very similar to his finite temperature counterpart. Instead a very different behavior would arise if aging was due to activated hopping over (free-) energy barriers. The interpretation is exactly the same outlined in the previous section on coarsening except that now the eigenvectors of the Hessian change during the time

\footnotetext{
${ }^{6}$ In reality only at zero temperature this interpretation is fully justified because otherwise the dynamics cannot be mapped exactly into an evolution within the free-energy landscape.
} 

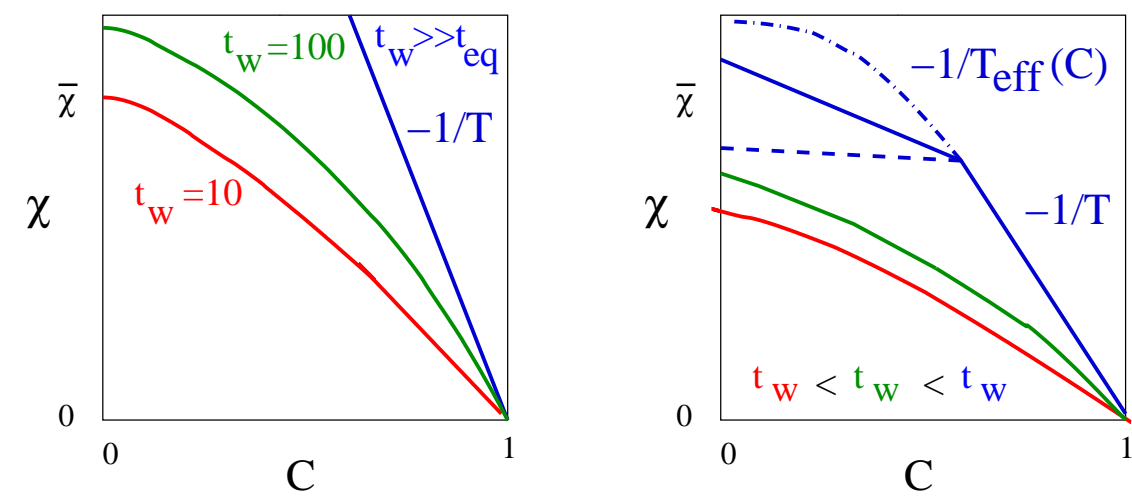

Figure 5: The asymptotic behavior of the integrated linear response against the correlation in a parametric plot, for fixed waiting time and using $t$ as a parameter. Left: behavior for a system relaxing to equilibrium. Right: behavior in a slowly relaxing system out of equilibrium. The dot-dashed line corresponds to a case with an infinite number of timescales $h(t)$ and $T_{\text {eff }}$. The continuous line with a breaking point corresponds to a systems, like the p-spin spherical model, with only one $T_{\text {eff }}$. The dotted line corresponds to the domain growth in the Ising model. Finally the other curves correspond to waiting times far from the asymptotic regime.

evolution. Another important new element with respect to domain growth in the Ising model is that for these mean-field glassy systems there are an exponential number (in the systems size) of thermodynamic states. It is generically believed and indeed it has been shown in simple cases that the system ends up, at long times, aging very close to the states that are marginally stable, i.e. that have a vanishing fraction of flat directions (at least after a very slow quench). Finally, in the case of discontinuous spin glasses, the effective temperature has been related to the complexity $S(f)$, i.e. the log degeneracy of states with a given free-energy $f$, as $1 / T_{\text {eff }}=\partial S / \partial f$ where the derivative is evaluated at the free energy density of the marginally stable states.

I will not continue further the discussion of the mean field theory of aging. I refer to more detailed review for deeper analysis [1] and to the conclusion for a general discussion.

\subsection{Activated dynamic and scaling}

Another way to tackle and describe the aging dynamics that has been developed in the last twenty years is based on a growing correlation length, scaling and renormalization group ideas. The physical picture that one has in mind is similar to the one discussed for the aging dynamics of the Ising model. After a quench to the low temperature phase domains separating the two (or more) phases grow. 
However, because of quench disorder, the domain walls have to overcome (free)energy barriers in order to grow. The scaling hypothesis is that at a timescale $t$ the typical linear domain size is $\xi(t)$ and that correlation functions and responses at different times can be written in terms of a unique scaling function up to a length rescaling. For example, as for the non disordered Ising model the spin-spin correlation function is expected to read:

$$
<\phi(\mathbf{x}, t) \phi\left(\mathbf{x}^{\prime}, t^{\prime}\right)>=f\left(\frac{\left|\mathbf{x}-\mathbf{x}^{\prime}\right|}{\xi(t)}, \frac{\xi(t)}{\xi\left(t^{\prime}\right)}\right) .
$$

On this lengthscale, $\xi(t)$, the typical (free-)energy barrier that domains have to overcome in order to grow is assumed to scale like $\xi^{\psi}$. As a consequence, using the Arrhenius law relating time and energy barrier one finds that $\xi(t) \propto(T \log t)^{1 / \psi}$, i.e. a very slow activated aging dynamics. Note that the previous expression is correct only in the regime $\frac{\xi(t)}{\xi\left(t^{\prime}\right)} \neq 1$. Also in this case one expects a pseudoequilibrium regime, corresponding formally to $t, t^{\prime}>>1$ with $\frac{\xi(t)}{\xi\left(t^{\prime}\right)}=1$ and physically to the equilibrium relaxation in the regions inside the domains. A systems in which this description is believed to be correct is the Random Field Ising model, RFIM, (ferromagnetic Ising model with independent random fields on each site). This system has a ferromagnetic phase transition for a not too high value of the variance of the random fields (their mean is zero). After a quench at low temperature domains separating the positive magnetization phase from the negative magnetization phase grows following the laws discussed above. This description of the aging dynamics has been proposed for many different disordered systems like disordered ferromagnets, spin glasses, etc.... In some cases, like for spin-glasses, this description of the aging dynamics is still matter of debate. Notice also that, at least superficially, it is very different from the mean-field one described in the previous section which is not based at all on activated dynamics. In the following I shall present a sketchy analysis of the off-equilibrium dynamics of the 1d RFIM [7]. This model is an instructive example because the above heuristic predictions can be indeed obtained analytically and their physical content can be easily grasped. The Hamiltonian is:

$$
H=-J \sum_{i=1}^{N-1} s_{i} s_{i+1}-\sum_{i=1}^{N} h_{i} s_{i}
$$

where $h_{i}$ are independent, say Gaussian, random variables with zero mean and variance $g=\overline{h_{i}^{2}}$. At zero temperature, the Imry-Ma argument implies that the region of + and - have a typical size $L_{I M}=4 J^{2} / g$. The reason is simple: over a length $l$ the energy gain obtained aligning the spins with the random fields is $\propto-\sqrt{g l}$ whereas the energy loss for creating a domain wall between a region of + and - spins is $2 J$. These two contributions match precisely at $l=L_{I M}$. Thus, we find that the RFIM in one dimension has of course no phase transition; however as long as the typical distance between domains during aging is less than $L_{I M}$ 
(or its finite-temperature counterpart) the system does not know it and the aging behavior is expected to be described by the activated dynamics scaling scenario described before. Note that this is also an example of interrupted aging in which if one waits long enough the system eventually equilibrates.

The way to tackle the aging dynamics of the 1D RFIM is to rewrite its Hamiltonian in terms of positions of domain walls. Let me call $a$ and $b$ respectively the domains $+\mid-$ and $-\mid+, N_{a}$ and $N_{b}$ their number, and $a_{i}$ and $b_{i}$ the position of the ith domain of type $a$ and $b$. Using this variables one can write the Hamiltonian as

$$
H=-J(N-1)-\sum_{i=1}^{N} h_{i}+2 J\left(N_{a}+N_{b}\right)+\sum_{i}^{N_{a}} V\left(a_{i}\right)-\sum_{i}^{N_{b}} V\left(b_{i}\right)
$$

where $V(x)=-2 \sum_{i=1}^{x} h_{i}$. Furthermore, a Glauber dynamics on the spins (i.e. with a transition rate $\left.w\left(s_{i} \rightarrow-s_{i}\right)=e^{-\beta \Delta E} /\left(e^{-\beta \Delta E}+e^{+\beta \Delta E}\right)\right)$ implies a reactiondiffusion dynamics for the domains in which a couple domains can be created on two neighboring sites with energy cost $4 J \pm h_{i}$, diffusion takes place with an energy cost $\pm 2 h_{i}$ and the domains annihilate on neighboring sites with an energy cost $-4 J \pm h_{i}$. We are interested in the aging dynamics after a quench to a very low temperature $T<<J$ and with $g<<J^{2}$. This is the case in which $L_{I M}>>1$ and the activated dynamics scaling picture should hold. In this case the effective dynamics for the domains is: $a$ domains diffuse within the external potential $V$, $b$ domains diffuse within the external potential $-V$ and $a$ and $b$ annihilates when they meet. The activated dynamics scaling picture now becomes very concrete. At time $t$ domains are at a typical distance $\xi(t)$. This length is just the typical distance $d_{S}(t)$ on which a particle diffuses in the interval of time $t$ in the random potential $V$ (or $-V$, the sign does not matter). The main reason is that on lengthscales smaller than $d_{S}(t)$ there should be just one domain because if there were others then they would have meet and therefore they have been annihilated on timescale $t$. Instead on lengthscales larger than $d_{S}(t)$ all the domains that were present before the quench are still there, thus $\xi(t)=d_{S}(t)$.

The problem of determining $d_{S}(t)$ and analyzing the motion before annihilation of $a$ and $b$ 'particles' is very well-known because is a discrete version of the Sinai Model: a particle diffusing with a Langevin dynamics in a Gaussian independent random force field. Indeed integrating the force to get the potential one finds precisely $V(x)=\sum_{i=1}^{x} f_{i}$ where $f_{i}$ are independent Gaussian random variables. It has been proved by Sinai that $d_{S}(t) \propto(T \log t)^{2}$. There is also a simple argument to heuristically get that $\psi=2$ : the potential $V(x)$ in an interval of length $d$ is like a Brownian motion (in which $V$ is the position and $x$ the time), thus the typical excursion of $V$ around its boundary values is $\sqrt{d}$. Hence, using the Arrhenius law we get precisely $d_{S}(t) \propto(T \log t)^{2}$.

Finally, the other nice thing about the dynamics of the 1D RFIM is that it can be really fully analyzed within a real space renormalization group procedure that provides an analytical derivation of the activated dynamics scaling picture and 
of many other results [7]. This RG procedure has been introduced by Dasgupta and $\mathrm{Ma}$ and for disordered quantum spin chains and it has been developed and put on a firmer theoretical ground by D.S. Fisher and subsequently applied to many different systems 8 .

I will not continue further the presentation of this very appealing scenario for the aging dynamics and I refer to reviews for other details [8, 1] and to the conclusion for a further discussion.

\subsection{Trap Model}

The trap model for aging dynamics has been introduced by Bouchaud in [9] and extensively developed since then 11. The trap model is not a microscopic model. It is however very interesting because on the one hand it has been used and it is still used a lot on a phenomenological level to interpret results of experiments and simulation and, on the other hand, it provides a coarse grained description of different microscopic models.

The simplest version [9] is defined as follows: there are $N$ possible states plotted as wells in Fig. [6. The dynamical evolution is determined by stochastic jumps: each well acts as a trap for the system. The trapping time is determined by the Arrhenius law: $\tau_{\alpha}=\tau_{0} \exp \left(+\left(F_{0}-F_{\alpha}\right) / T\right)$ where $F_{\alpha}$ is the free energy of the "state" (or well, trap) $\alpha$ and $F_{0}$ is a threshold free-energy state, see Fig. [6. Once

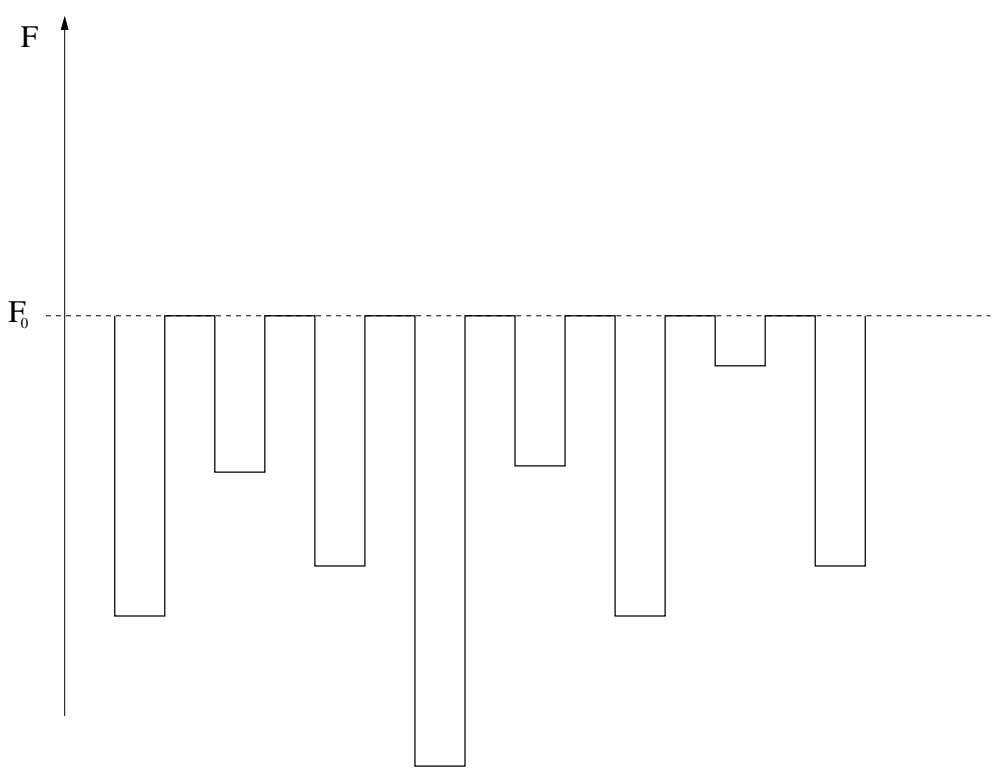

Figure 6: Schematic representation of the configuration space for the trap model.

the system is escaped from a trap, it falls completely at random into another 
trap. The rationale behind these rules is that generically for disordered systems there exists many different metastable states which, at least in some regimes, are surrounded by large energy barriers. The complete absence of geometry is of course the simpler assumption one can do. More complicated structures of the configuration space has been considered. The other essential ingredient of the model is the distribution of free-energies that is taken exponential with a certain parameter $x$ :

$$
P(F)=x / T \exp \left(x\left(F-F_{0}\right) / T\right) .
$$

This choice is motivated by the presence of a similar distribution in mean-field disordered systems like the Random Energy Model (in which $x=T / T_{g}$ ) [1] and, on general grounds, by the extreme value statistics for deep free-energy states [1. The resulting distribution of trapping times $\psi(\tau)$ reads:

$$
\psi(\tau)=x \frac{\tau_{0}^{x}}{\tau^{1+x}}
$$

The important feature of this law is that the average trapping time $\langle\tau\rangle$ is infinite for $x<1$, i.e. $T<T_{g}$ if we take $x$ as proportional to the temperature. In this regime the system, taken at time zero uniformally distributed over the traps, never reaches the equilibrium and ages forever. In this case the nature of the aging dynamics is particularly clear [9]: the time $t_{w}$ after $n$ jumps equals $\sum_{i=1}^{n} \tau_{i}$ where $\tau_{i}$ are independent variables with probability law $\psi(\tau)$. It is a well-known result that since the first moment is infinite the central limit theorem does not apply, $t_{w}$ is distributed with a Levy Law and its typical value is of the order of the maximum trapping time encountered during the evolution. At a given waiting time $t_{w}$ the sum $\tau_{1}+\tau_{2}+\ldots$ is dominated by its largest term which is of the same order of $t_{w}$. In this case it is very clear that the system ages because the relaxation time is set by the age of the system itself.

I will not present further results on the trap model and I refer to [9, 1] for a wider presentation. I would like just to stress that the Trap Model provides a coarse grained description of the Sinai model in a biased potential [8] as well as the aging dynamics (on extremely long times, diverging with the system sizes) of the Random Energy Model [10]. These results suggest that it is not only a phenomenological model and it might play in the future an important role in the description of the finite dimensional extension of the mean-field aging.

\section{$5 \quad$ Outstanding issues}

In this section I would like to point out what are in my mind some outstanding theoretical issues in aging of glassy systems. It will be certainly amazing to look at this list in a couple of years to see how things turned out to be very different. Beyond the mean-field theory of Aging. Length-scales, Landau Theory and fluctuations. As for critical phenomena one 
can identify three steps that have to be followed to analyze the physics of finite dimensional systems. First, one can solve an infinite dimensional system. This has been done for aging and these studies indeed lead to a mean-field theory of aging [1. A second important step is set up a Landau theory. Although the results still are mean-field like (i.e. no fluctuations are taken into account) this provides hints of why results might be universal (at least in higher enough dimension) of which type of correlation lengths are diverging (as we discussed in the introduction if the aging is not interrupted one expect a diverging length) and it helps to clarify what are the important fluctuations that one has to take into account. I think that it is a very important point that will be studied in detail in the future, some results have been already obtained in 11.

Beyond mean-field theory: activated processes. For mean-field systems conjectured to be related to structural glasses the thermodynamic as well as the equilibrium and off-equilibrium dynamics are expected to change drastically in finite dimension. The main reasons is that the physics of these models is dominated by the existence of an infinite number of thermodynamic states and, instead, it is clear that in finite dimension this exponential proliferation of thermodynamic states cannot take place. How this is going to change the physics (statics and, specially, dynamics)? How this translate in terms of real space structure? What remains of the off-equilibrium dynamics described in terms of energy landscape picture? These are certainly very important and pressing questions that, hopefully, will receive a lot of attention in the near future.

Renormalization group for the activated dynamic scaling.

Although in one dimension there are examples of activated dynamic scaling that can be derived and fully analyzed analytically within a RG procedure the extension to higher dimensions remains an important open problem and certainly a major challenge for the future. Recent interesting results have been already obtained in [12, 13].

Generalization of the Fluctuation-Dissipation relation in finite dimension

This question has already attracted a lot of attention. In particular one would like to know if and how the FDR is generalized in finite dimension in comparison with mean-field predictions and if the resulting $T_{\text {eff }}$ can be really interpreted as an effective temperature. Furthermore also the generalization of FDR to driven off-equilibrium systems as for example granular media is an interesting related question. Despite many simulations and some experiments have been done these questions are not completely settled and certainly they will continue to give rise to many works in the future.

\section{Aging in glassy systems without quenched disorder}

There are systems like structural glasses that have a very interesting slow dynamics that is still a puzzle despite many years of theoretical and experimental investigations. The understanding of their off-equilibrium dynamics is therefore a challenge for the future. 
From this point of view it is very likely that in the future much more attention will be devoted to the off-equilibrium dynamics of simple statistical mechanics model of glassy systems as Kinetically Constrained Models, Lattice Glasses, ... (see [14] for a review).

Quantum off-equilibrium dynamics What about quantum glassiness? This is certainly a very interesting subject that will probably be the target of many investigations in the future. To me very interesting questions are: what are the properties of off-equilibrium dynamic (aging) close to a quantum critical point? What are the properties of the aging behavior induced by a quench (that goes through a quantum phase transition) due to a sudden change in the control parameter governing quantum fluctuations? What is a quantum glass without disorder?

Some of these questions have been recently addressed in [16, 15].

I will stop here this list that could go on much further. I think I have described already enough challenging problems.

\section{$6 \quad$ Suggested readings}

Suggested readings that are useful to have a deeper understanding of the aging dynamics and the theoretical ideas developed to understand it are:

J. Kurchan and L. Laloux, J. Phys. A 29, 1929 (1996) for the energy descent picture of aging.

L. F. Cugliandolo and J. Kurchan, Phys. Rev. Lett. 71, 173 (1993) for the aging dynamics in mean field glassy systems.

D. S. Fisher and D. A. Huse, Phys. Rev. B 38, 373 (1988), Phys. Rev. B 35, 6841 (1987) for the activated dynamic scaling.

J.-P. Bouchaud, J. Phys. I France 2, 1705 (1992) for the Trap model.

This list is clearly not exhaustive.

\section{Acknowledgments}

I thank the organizers of the school and conference "Unifying Concepts in Glass Physics III" for the invitation to give these lectures and C. Monthus for a careful reading of this manuscript. Along the years I learned a lot about aging dynamics from many colleagues and in particular L.F. Cugliandolo and J. Kurchan that I am very glad to thank. Finally I acknowledge partial support from the European Community's Human Potential Programme contracts HPRNCT-2002-00307 (DYGLAGEMEM). 


\section{References}

[1] J.-P. Bouchaud, L. Cugliandolo, J. Kurchan, M. Mézard, in Spin-glasses and Random Fields, edited by A. P. Young (World Scientific, Singapore, 1998).

L. Cugliandolo, in Slow relaxations and non-equilibrium dynamics in condensed matter, Les Houches, Session LXXVII, J. L. Barrat, M. Feigelman, J. Kurchan, J. Dalibard Edts, Springer-EDP Sciences (2003).

J.-P. Bouchaud in Soft and Fragile Matter, M.E. Cates and M. Evans Edts, IOP (2000).

[2] E. Vincent, M. Hammann, M. Ocio, J.-P. Bouchaud, L. F. Cugliandolo cond-mat/9607224 Proceedings of the Sitges conference (E. Rubi ed, Springer-Verlag, 1997).

[3] A. J. Bray, Adv. in Phys. 43 (1994) 357.

[4] J. Kurchan and L. Laloux, J. Phys. A 29, 1929 (1996).

[5] A. Cavagna, Lecture notes in the same volume.

[6] D.R. Reichman, Lecture notes in the same volume.

[7] D. S. Fisher, P. Le Doussal, C. Monthus, Phys. Rev. E 64, 066107 (2001).

[8] For a review see Strong disorder $R G$ approach of random systems C. Monthus and F. Igloi, cond-mat/0502448, Phys. Rep. in press.

[9] J.-P. Bouchaud, J. Phys. I France 2, 1705 (1992).

[10] G. B. Arous, A. Bovier, and V. Gayrard Phys. Rev. Lett. 88, 087201 (2002).

[11] C. Chamon, M. P. Kennett, H. Castillo, L. F. Cugliandolo, Phys. Rev. Lett. 89217201 (2002); H. E. Castillo, C. Chamon, L. F. Cugliandolo, M. P. Kennett, Phys. Rev. Lett. 88, 237201 (2002).

[12] L. Balents and P. Le Doussal, EuroPhys. Lett. 65685 (2004) and condmat/0408048 to appear in Adv. in Physics.

[13] G. Schehr, P. Le Doussal, Functional Renormalization for pinned elastic systems away from their steady states, condmat/0501199; Phys. Rev. Lett. 93, 217201 (2004).

[14] F Ritort and P Sollich, Adv. in Phys. 52219 (2003).

[15] C. Chamon, Phys. Rev. Lett. 94, 040402 (2005).

[16] H. Westfahl Jr., J. Schmalian, P. G Wolynes, Phys. Rev. B 68, 134203 (2003). 